\title{
Rare case of vaginal leiomyoma in an adolescent girl: a diagnostic dilemma
}

\author{
Manisha Upadhyay ${ }^{1}$, Meenakshi Chauhan ${ }^{1 *}$, Sarika Gautam ${ }^{1}$, Tek Chand Yadav $^{2}$
}

\begin{abstract}
${ }^{1}$ Department of Obstetrics and Gynaecology, ${ }^{2}$ Department of Medicine, Pandit Bhagwat Dayal Sharma, Post Graduate Institute of Medical Sciences, Rohtak, Haryana, India
\end{abstract}

Received: 06 January 2018

Accepted: 31 January 2018

\section{*Correspondence:}

Dr. Meenakshi Chauhan,

E-mail: mbc51490@yahoo.co.in

Copyright: (C) the author(s), publisher and licensee Medip Academy. This is an open-access article distributed under the terms of the Creative Commons Attribution Non-Commercial License, which permits unrestricted non-commercial use, distribution, and reproduction in any medium, provided the original work is properly cited.

\begin{abstract}
Vaginal leiomyoma are very uncommon primary tumors of vagina. They are extremely rare in adolescent age group. They can have variable clinical presentations and could be difficult to diagnose at times in adolescents. We report an extremely rare and novel case of primary vaginal leiomyoma with hyaline degeneration in an adolescent girl which was primarily misdiagnosed as hematocolpos or vaginal malignancy due to its clinical presentation. A 14-year-old girl presented with complains of difficulty in passing urine and irregular vaginal bleeding. On per rectal examination an ill-defined bulge was felt anteriorly and altered chocolate color blood was coming out of vagina. Her ultrasonography report was suggestive of a cervicovaginal mass or hematocolpos. So, examination under anesthesia (EUA) was planned with probable diagnosis of hematocolpos due to secondary reasons or malignancy. On EUA a soft cystic vaginal mass was felt arising from the anterolateral wall obscuring the cervical os and cervix was felt behind it separately. Biopsy was taken from the mass which was confirmed histopathologically and immunohistochemically as vaginal leiomyoma with hyaline degeneration. Patient was managed successfully with complete tumor resection through vaginal approach followed by vaginal reconstruction. Though rare but the differential diagnosis of vaginal leiomyoma should always be kept in mind in adolescent girls presenting with vaginal mass and abnormal vaginal bleeding. Primary vaginal leiomyoma can be completely cured by surgery if diagnosed timely and appropriately so, should never be missed in adolescent age group.
\end{abstract}

Keywords: Adolescent girl, Vaginal leiomyoma

\section{INTRODUCTION}

Uterine leiomyoma is a very common benign tumor of the female genital tract but unusually leiomyoma can also arise from adnexa, cervix, vulva, and vagina. Vaginal leiomyoma are very rare primary tumors of vagina with approximately 300 cases reported so far. ${ }^{1}$

The earliest reference made of such a tumor is attributed to De Leyden in 1773, and the first review of literature concerning such tumors was published in $1882 .^{2}$ They usually arise from anterior vaginal wall and rarely from the posterior wall. Vaginal leiomyoma can be asymptomatic or can present with varied clinical features mimicking prolapse, urinary obstruction, vaginal bleeding, dysuria, dyspareunia, lower abdominal pain. ${ }^{3}$

Due to varied clinical presentation they have a wide spectrum of differential diagnosis and many times also get misdiagnosed.

Vaginal leiomyoma is extremely rare in the adolescent age group. We report a novel and rare case of vaginal fibroid in an adolescent girl which was assumed preoperatively to be a secondary hematocolpos or vaginal malignancy due to its unusual presentation. 


\section{CASE REPORT}

A 14-year-old adolescent girl presented to the outpatient department with complaints of difficulty in passing urine, irregular vaginal bleeding and hematuria for past 2-3 months. She used to feel incomplete evacuation and increase urge of passing urine. There was no preceding amenorrhea. She had menarche 2 years back. Her menstrual cycles were regular and used to continue for 67 days but for last three months she was having irregular intermenstrual vaginal bleeding. She had history of twounit blood transfusion 1 month back. On examination patient was extremely pale but the vitals were stable. Abdomen was soft with no palpable swelling. On local examination of genitalia a normal vaginal opening was seen and chocolate brown color blood was coming through it. On per rectal examination an ill-defined bulge was felt anteriorly. Patient was admitted with the clinical diagnosis of hematocolpos due to some secondary reasons or some vaginal malignancy. Her hemoglobin was $2.6 \mathrm{gm} / \mathrm{dl}$ and urine microscopic examination was not suggestive of any RBC's in urine. Her urine pregnancy test was negative. Thyroid profile was normal. Laboratory tests for blood dyscrasias were unremarkable. Ultrasonography report was suggestive of a heteroechoic cervicovaginal mass of $10 \times 7.3 \mathrm{~cm}$ showing flow on color Doppler. Kidney, bladder, uterus and adnexa were normal. MRI (Figure 1 and Figure 2 was suggestive of a soft tissue mass of $10 \times 8.9 \times 8 \mathrm{~cm}$ in vagina. There was evidence of hemorrhage and necrosis in the mass. The anterior vaginal wall was extremely thinned out but the mass was contained within the vagina. Mass was pushing bladder anterolaterally to right side and superiorly Uterus was normal. Bilateral ovaries were normal. There was no free fluid.

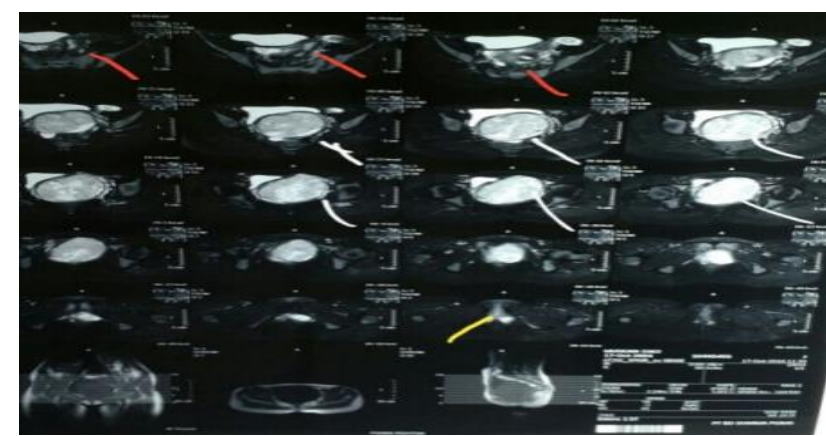

Figure 1: Axial STIR image showing a well-defined hyperintense mass (white marker) arising from anterior vaginal wall (yellow marker) with hypointense capsule around it.

No abnormality detected on cystoscopy. Her anemia was corrected by blood transfusion and examination done under anesthesia. On per speculum examination a bulge was seen in the anterior vaginal wall as shown in Figure 3. Per vaginal examination revealed a soft cystic vaginal mass which was felt arising from left anterolateral wall of vagina and obliterating whole of the vagina. Mass was obscuring the cervical os completely and cervix was felt high up behind it separately. The dark chocolate brown color blood was seen coming through the cervical opening. Urethra was also seen receded back which on catheterization drained clear urine. Biopsy was taken from that mass which was confirmed histopathologically and immunohistochemically as vaginal leiomyoma.

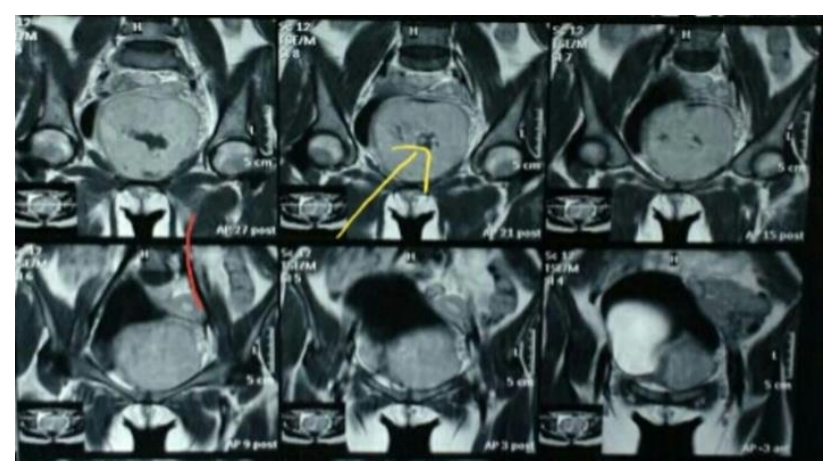

Figure 2: Coronal T1 image showing well defined hyperintense mass (yellow marker) with areas of hemorrhage and necrosis in the centre.

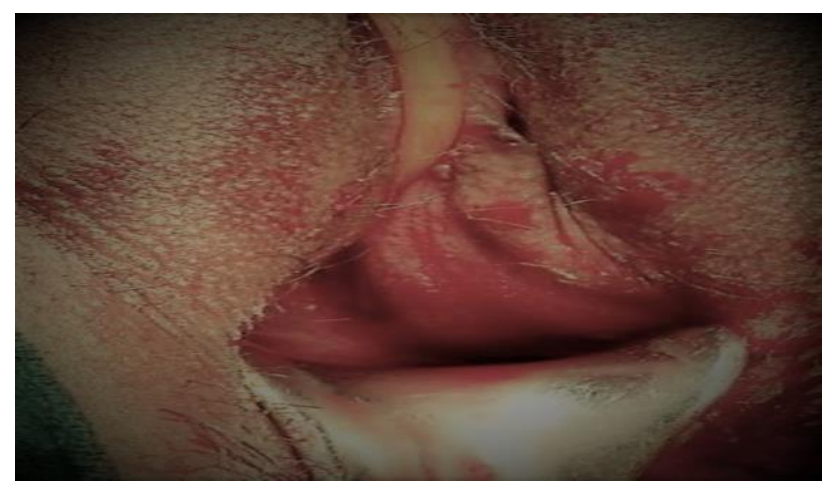

Figure 3: Per speculum view showing the myoma bulge in anterior vaginal wall.

Patient planned for vaginal myomectomy and complete resection but the tumor could be resected in piece meal due to its extremely soft consistency (Figure 4).

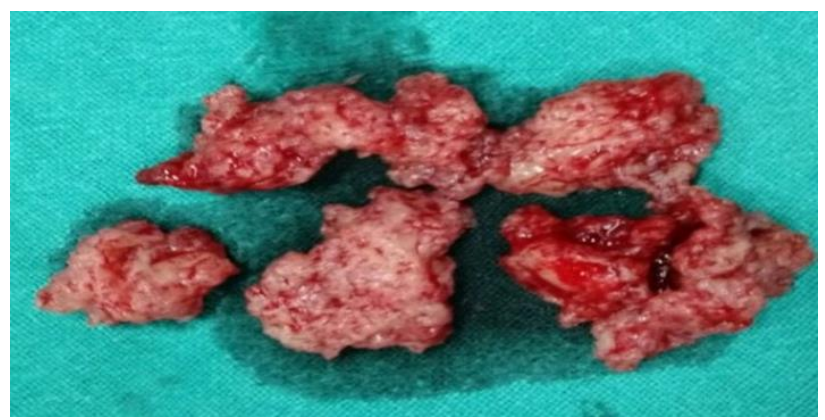

Figure 4: Specimen of vaginal myoma removed in piece meal.

Resection of myoma was followed by vaginal reconstruction. The specimen enucleated was confirmed 
histopathologically as vaginal leiomyoma with areas of necrosis and hyaline degeneration. Postoperative period was uneventful and the patient was discharged in stable condition.

\section{DISCUSSION}

Vaginal tumors are rare and include papilloma, hemangioma, mucus polyp, and rarely leiomyoma. Leiomyoma of the female genital tract occur commonly in uterus and to some extent in the cervix followed by the round ligament, utero-sacral ligament, ovary, and inguinal canal. Occurrence in vagina is very rare. ${ }^{4}$ This rare site of occurrence of leiomyoma makes our case unique. There are very few cases of primary vaginal leiomyoma reported so far in the literature. Another uniqueness of our case is the age of our patient. Vaginal leiomyoma are commonly seen in the age group ranging from 35 to 50 years. ${ }^{5}$ Our patient was an adolescent which is the rare age for vaginal leiomyoma to occur.

Vaginal leiomyoma are usually firm in consistency but sometimes their consistency may vary to soft cystic due to degenerative changes. This may lead to various misdiagnosed differentials like cystocele, urethrocele, skene duct abscess, gartner duct cyst, urethral diverticulum, vaginal cyst, bartholin gland cyst, epidermal inclusion cyst and a variety of both benign and malignant vaginal tumors. ${ }^{5}$ Same was the case in our patient. Soft consistency of myoma due to hyaline degeneration leads to suspicion of some malignancy and the another misleading finding of chocolate brown color blood coming through the vagina was might be due to incomplete drainage of menstrual blood due to vaginal myoma obscuring the cervical os.

Vaginal myomas are usually firm, solitary and usually arise in the anterior wall but may be found in any location. They generally vary in size from 1 to $5 \mathrm{~cm}$ in diameter, and may or may not be pedunculated and covered mostly with smooth intact vaginal mucosa which differentiates it from malignancy where invasion of surrounding could be seen. They usually are asymptomatic until they reach a certain size (usually $\geq 6$ $\mathrm{cm}$ ). Liu analyzed 11 vaginal leiomyoma, and their average time to be symptomatic was 8.4 years. ${ }^{6}$ It can present variably depending upon the site, size, extent and presence of any degenerative change. In our case it presented with irregular vaginal bleeding which is due to the necrosis and obstructive urinary symptom due to its location in anterior vaginal wall.

Diagnosis can be made preoperatively with ultrasonography but MRI is better choice. ${ }^{7}$ Gold standard for diagnosis is histopathology. In MRI they appear as homogenous lesion with signal similar to that of myometrium. $^{7}$ MRI especially provides useful preoperative information about the morphological and structural characteristics of the mass and the anatomical relationship between the lesion. ${ }^{8}$ A vaginal leiomyoma generally has low signal intensity on T1-weighted images similar to surrounding muscles and still relatively low signal intensity on T2-weighted images. ${ }^{7-9}$ while malignant tumors generally have higher signal intensity on T2 images with irregular margins and most are heterogeneous enhanced after gadolinium administration due to the presence of areas of necrosis or hemorrhage. ${ }^{8}$ But degenerative changes in myoma can also show high signal intensity changes misleading to diagnosis of malignancy as was the case in our patient.

Definitive treatment is always surgical. Enucleation and excision is the treatment of choice. Approach should be selected wisely. This is either by the abdominal or vaginal route, depending on the location of the tumor. ${ }^{10}$ Recurrence has also been seen. Hence the patient needs to be followed up.

Clinical significance: Primary vaginal leiomyoma can be readily and completely cured by surgery if diagnosed timely and appropriately but they are often misdiagnosed due to its variable clinical presentation. So, the diagnosis should always be kept in mind while examining an adolescent patient with vaginal mass or abnormal vaginal bleeding.

\section{CONCLUSION}

Vaginal leiomyoma though rare can have variable and misleading clinical presentations in adolescent age group as it presented with urinary and menstrual complains in our case and can often mimics malignancy. A thorough clinical examination will direct towards the right diagnosis which could be confirmed radiologically and histopathologically. Surgery is the definitive treatment.

\section{ACKNOWLEDGMENTS}

Authors would like to thank the patient for consenting to reveal the case details and photographs for publication.

Funding: No funding sources

Conflict of interest: None declared

Ethical approval: Not required

\section{REFERENCES}

1. Chakrabarti I, De A, Pati S. Vaginal Leiomyoma. J Midlife Health. 2011;2(1):42-3.

2. Gottwald L, Welfel J, Akoel KM, Lech W, Suzin J, Bieńkiewicz A. Vaginal leiomyoma. Ginekol Pol. 2003;74(3):224-6.

3. Elsayes KM, Narra VR, Dillman JR, Velcheti V, Hameed $\mathrm{O}$, Tongdee $\mathrm{R}$, et al. Vaginal masses: magnetic resonance imaging features with pathologic correlation. Acta Radiol. 2007;48(8):921-33.

4. Young SB, Rose PG, Reuter KL. Vaginal fibromyomata: two cases with preoperative assessment, resection and reconstruction. Obstet Gynecol. 1991;78:972-4. 
5. Bennett HG, Erlich MM. Myoma of the vagina. Am J Obstet Gynecol. 1941;42:314-20.

6. Liu MM. Fibromyoma of the vagina. Eur J Obstet Gynecol Reprod Biol. 1988;29(4):321-8.

7. Shadbolt CL, Coakley FV, Qayyum A, Donat SM. MRI of vaginal leiomyomas. J Comput Assist Tomogr. 2001;25(3):355-7.

8. Pavlica P, Bartolone A, Gaudiano C, Barozzi L. Female paraurethral leiomyoma: ultrasonographic and magnetic resonance imaging findings. Acta Radiol. 2004;45(7):796-8.

9. Ikeda R, Suga K, Suzuki K. MRI appearance of a leiomyoma of the female urethra. Clin Radiol 2001;56(1):76-9.
10. Tramier D, Marinetti C, Jouve MP. Leiomyoma of the vagina: a report on 2 cases author's transl. J Gynecol Obstet Biol Reprod (paris). 1980;9(3):3678.

Cite this article as: Upadhyay M, Chauhan M, Gautam S, Yadav TC, Pandey A. Rare case of vaginal leiomyoma in an adolescent girl: a diagnostic dilemma. Int J Reprod Contracept Obstet Gynecol 2018;7:1269-72. 